\title{
SSTR3 is a putative target for the medical treatment of gonadotroph adenomas of the pituitary
}

\author{
Misu Lee, Amelie Lupp', Nigel Mendoza ${ }^{2}$, Niamh Martin ${ }^{3}$, Rudi Beschorner ${ }^{4}$, \\ Jürgen Honegger ${ }^{5}$, Jürgen Schlegel ${ }^{6}$, Talia Shively $^{3}$, Elke Pulz, Stefan Schulz ${ }^{1}$, \\ Federico Roncaroli ${ }^{3}$ and Natalia S Pellegata
}

Institute of Pathology, Helmholtz Zentrum München, D-85764 Neuherberg, Germany

${ }^{1}$ Department of Pharmacology and Toxicology, Jena University Hospital, Friedrich Schiller University, Jena, Germany

${ }^{2}$ Departments of Neurosurgery ${ }^{3}$ Medicine, Imperial College, St Dunstans Road, London W6 8RP, UK

${ }^{4}$ Department of Neuropathology, Institute for Pathology and Neuropathology ${ }^{5}$ Department of Neurosurgery, University of Tübingen, Tübingen, Germany

${ }^{6}$ Institute of Pathology, Technical University of Munich, München, Germany
Correspondence should be addressed to N S Pellegata; F Roncaroli Emails

natalia.pellegata@ helmholtz-muenchen.de; f.roncaroli@imperial.ac.uk

\begin{abstract}
Gonadotroph pituitary adenomas (GPAs) often present as invasive macroadenomas not amenable to complete surgical resection. Radiotherapy is the only post-operative option for patients with large invasive or recurrent lesions. No medical treatment is available for these patients. The somatostatin analogs (SSAs) octreotide and lanreotide that preferentially target somatostatin receptor type 2 (SSTR2) have little effect on GPAs. It is widely accepted that the expression of specific SSTR subtypes determines the response to SSAs. Given that previous studies on mRNA and protein expression of SSTRs in GPAs have generated conflicting results, we investigated the expression of SSTR2, SSTR3, and SSTR5 (the main targets of available SSAs) in a clinically and pathologically well-characterized cohort of 108 patients with GPAs. A total of 118 samples were examined by immunohistochemistry using validated and specific MABs. Matched primary and recurrent tissues were available for ten patients. The results obtained were validated in an independent cohort of 27 GPAs. We observed that SSTR3 was significantly more abundant than SSTR2 $(P<0.0001)$ in GPAs, while full-length SSTR5 was only expressed in few tumors. Expression of SSTR3 was similar in primary and recurrent adenomas, was high in potentially aggressive lesions, and did not change significantly in adenomas that recurred after irradiation. In conclusion, low levels of expression of SSTR2 may account for the limited response of GPAs to octreotide and lanreotide. Given the potent anti-proliferative, pro-apoptotic, and anti-angiogenic activities of SSTR3, targeting this receptor with a multireceptor ligand SSA such as pasireotide may be indicated for potentially aggressive GPAs.
\end{abstract}

\section{Key Words \\ - somatostatin receptors \\ - gonadotroph adenoma \\ - aggressive adenoma \\ - pasireotide}

Endocrine-Related Cancer (2015) 22, 111-119

\section{Introduction}

Somatostatin receptors (SSTRs) are G protein-coupled molecules encoded by five distinct genes (SSTR1, SSTR2, SSTR3, SSTR4, and SSTR5) (Theodoropoulou \& Stalla 2013,
Cuevas-Ramos \& Fleseriu 2014). SSTR1, SSTR2, and SSTR3 are constitutively expressed in the normal human pituitary while SSTR4 and SSTR5 are expressed at a low level. 
In pituitary adenomas, expression of SSTRs varies among types and within each tumor type (Theodoropoulou \& Stalla 2013, Cuevas-Ramos \& Fleseriu 2014). When they bind to their natural ligands, somatostatin 14 and 28, SSTRs exert both a regulatory effect on secretion of pituitary hormones by inhibiting the synthesis of their mRNA and their release, and a potent anti-proliferative activity (Theodoropoulou \& Stalla 2013). For these reasons, SSTRs are established targets for peptide receptor therapy with somatostatin analogs (SSAs).

The SSAs octreotide and lanreotide bind mainly to SSTR2 and to a lesser extent to SSTR3 and SSTR5, and represent the mainstay of medical therapy of functioning somatotroph and thyrotroph adenomas (Ben-Shlomo \& Melmed 2008, Grozinsky-Glasberg et al. 2008, Theodoropoulou \& Stalla 2013). Treatment of acromegalic patients with these SSAs reduces or normalizes growth hormone and insulin-like growth factor 1 levels and induces tumor shrinkage (Theodoropoulou \& Stalla 2013). Owing to the restricted affinity of these two compounds for SSTRs, the multiligand SSA pasireotide was developed. Pasireotide has a 158-, > 30-, and 11-fold higher functional activity than octreotide on SSTR5, SSTR1, and SSTR3 respectively (Schmid \& Schoeffter 2004, Theodoropoulou \& Stalla 2013). Though not free of side effects, pasireotide has shown promising results in the treatment of acromegalic patients (Ben-Shlomo \& Melmed 2008) and, more recently, in the treatment of patients with Cushing's disease (Colao et al. 2012, Webb et al. 2014). A clinical trial led by Gadelha and colleagues is currently recruiting patients to evaluate the effect of pasireotide on regrowth of clinically non-functioning pituitary adenomas (NFPAs) (www.ClinicalTrials.gov; identifier NCT01620138).

Gonadotroph pituitary adenomas (GPAs) account for approximately $35 \%$ of all pituitary tumors. They are usually clinically nonfunctioning and are often diagnosed at the occurrence of signs and symptoms of mass effects (Young et al. 1996). From 30\% to $45 \%$ of GPAs extend into the cavernous sinus and fewer cases invade the sellar floor causing considerable morbidity to patients (Brochier et al. 2010). Large and invasive macroadenomas are not amenable to complete resection and can regrow in up to almost half the cases (Brochier et al. 2010, Berkmann et al. 2014). Radiotherapy is the only post-operative option for residual and recurrent lesions as no effective medical treatment is available. Indeed, previous studies have documented little efficacy of octreotide and lanreotide in NFPAs (Colao et al. 2011).

As the response of pituitary adenomas to SSAs depends on the expression of specific SSTR subtypes
(Gatto et al. 2013), and their level of expression may vary among tumors, assessment of SSTRs of tumor tissue might allow for a more accurate stratification of patients who may benefit from SSA therapy. Previous studies assessing expression of SSTR2 in GPAs have produced conflicting results (Pawlikowski et al. 2003, Pisarek et al. 2009, Ramirez et al. 2012). Therefore, we aimed to determine the expression profile of SSTR2, SSTR3, and SSTR5 in a large and homogeneous cohort of patients with primary or recurrent GPAs. As mRNA and protein levels of SSTRs do not always correlate in pituitary adenomas (Nielsen et al. 2001), we used immunohistochemistry (IHC) with specific and validated antibodies. The results obtained in our cohort of 108 patients were validated in an independent series of 27 GPA patients collected at different institutions.

Our results indicate that SSTR3 is the most abundant receptor subtype in GPAs. Atypical and recurrent GPAs show elevated expression of SSTR3, which is maintained after radiotherapy. SSTR3 could be the target of pharmacological treatment with pasireotide in patients with GPAs, particularly those with aggressive/ recurrent disease.

\section{Patients and methods}

\section{Patient selection and tumor samples (test cohort)}

From the Brain and Pituitary Tumor Registry at Imperial College, London, UK, we retrieved data for all patients who underwent transsphenoidal surgery (TSS) performed by the same neurosurgeon (N M) for GPA between January 2005 and December 2013. We identified 108 patients whose medical records and pre- and post-operative imaging were available for review. Patients with apoplexy were excluded. Seven patients were re-operated for recurrent disease within the study period and 11 of the 108 adenomas were recurrent lesions for which the primary tumor had been removed before 2005. Overall, the tissue from the primary and recurrent adenoma was available for examination in ten cases. Six patients showed measurable tumor regrowth at follow-up neuroimaging but were not re-operated on. The clinical characteristics of these patients are summarized in Table 1 .

A total of 118 adenoma samples (108 consecutive cases and the corresponding primary tumors of ten recurrences) were assessed for expression of SSTRs. All tumors except two were clinically nonfunctioning. Representative paraffin blocks were selected for SSTR IHC using the original hematoxylin and eosin (H\&E)-stained

Published by Bioscientifica Ltd 
Table 1 Essential clinical and pathological features of the GPAs in our test cohort. Total number of patients: 108 (73M/35F). Age: mean years (range), 56 (24-84); males 58 and females 53

\begin{tabular}{|c|c|}
\hline & Percentage \\
\hline \multicolumn{2}{|l|}{ Signs and symptoms at onset (patients) } \\
\hline Visual impairment & $75.9 \%(82 / 108)$ \\
\hline Hypopituitarism & $20.4 \%(22 / 108)$ \\
\hline Raised prolactin & $11.1 \%(12 / 108)$ \\
\hline Incidentalomas & $8.3 \%(9 / 108)$ \\
\hline Headache & $6.5 \%(7 / 108)$ \\
\hline Amenorrhea & $5.6 \%(6 / 108)$ \\
\hline Gynecomastia & $0.9 \%(1 / 108)$ \\
\hline \multicolumn{2}{|l|}{ Extension/invasion (tumors) } \\
\hline Extension into cavernous sinus & $25 \%(27 / 108)$ \\
\hline Bone invasion & $13.0 \%(14 / 108)$ \\
\hline \multicolumn{2}{|l|}{ Follow-up } \\
\hline Mean months (range) & $45(7-110)$ \\
\hline Regrowth & $5.5 \%(6 / 108)$ \\
\hline Re-operation & $6.5 \%(7 / 108)$ \\
\hline Recurrence $^{a}$ & $16.7 \%(18 / 108)$ \\
\hline Post-operative radiotherapy & $17.6 \%(19 / 108)$ \\
\hline \multicolumn{2}{|l|}{ Pathological features (tumors) } \\
\hline \multicolumn{2}{|l|}{ Mitotic activity } \\
\hline Absent & $60.2 \%(65 / 108)$ \\
\hline Present (range $1 \times 50-20 \times 50 \mathrm{HPF}$ ) & $39.8 \%(43 / 108)$ \\
\hline \multicolumn{2}{|l|}{ Immune profile } \\
\hline $\mathrm{FSH} \beta$ & $88.9 \%(96 / 108)$ \\
\hline $\mathrm{LH} \beta$ & $43.5 \%(47 / 108)$ \\
\hline$\alpha \mathrm{GSU}$ & $75 \%(81 / 108)$ \\
\hline \multicolumn{2}{|l|}{ Others } \\
\hline Oncocytic changes & $13.8 \%(15 / 108)$ \\
\hline \multicolumn{2}{|l|}{ Ki-67 } \\
\hline$<3 \%$ & $89.8 \%(97 / 108)$ \\
\hline$\geq 3 \%$ & $10.2 \%(11 / 108)$ \\
\hline \multicolumn{2}{|l|}{$\mathrm{p} 53$} \\
\hline Negative & $92.6 \%(100 / 108)$ \\
\hline Positive & $7.4 \%(8 / 108)$ \\
\hline Atypical (WHO, ref. Lloyd et al. 2004) & $5.6 \%(6 / 108)$ \\
\hline Grade $2 b$ (ref. Trouillas et al. 2013) & $6.5 \%(7 / 108)$ \\
\hline
\end{tabular}

HPF, high-power field.

a Seven patients recurred within the study period; the other 11 patients presented with recurrent lesions whose primary tumors were removed before 2005 .

sections. The original H\&E-stained sections and immunostains for anterior pituitary hormones, MIB1 (Ki-67), and p53 were reviewed (Table 1). Steroidogenic factor 1 was also analyzed in all cases to further confirm the diagnosis of GPAs. Mitotic activity was evaluated in 100 fields at a magnification of $\times 40$ (Nikon Plan Fluor $\times 0.75$, Nikon GmbH, Düsseldorf, Germany). A total of 65 adenomas did not show any mitoses. The remaining 43 adenomas showed a mitotic count ranging between $1 \times 50$ and $20 \times 50$ high-power fields. Immunostaining for Ki-67 was performed on all 118 tumor samples. The Ki-67 labeling index was calculated as the mean percentage of stained nuclei of tumor cells irrespective of intensities for
1000 cells in three representative fields (total 3000 cells). It was equal to or higher than $3 \%$ in 11 out of 108 cases $(10.2 \%)$. Six $(5.6 \%)$ were atypical adenomas defined according to the current WHO classification (Lloyd et al. 2004). When classified following the grading criteria proposed by Trouillas et al. (2013), 63 GPAs were grade $1 \mathrm{a}$, nine were $1 \mathrm{~b}, 39$ were $2 \mathrm{a}$, and $7(6.5 \%)$ were $2 \mathrm{~b}$. Pathological features of an example of grade $2 \mathrm{~b}$ adenoma are shown in Supplementary Figure 1, see section on supplementary data given at the end of this article. Oncocytic changes were observed in 15 out of 108 tumors (13.8\%). Tumor characteristics are summarized in Table 1.

These studies were approved by the ethical committees of the Imperial College and patients signed an informed consent.

\section{Independent validation cohort}

In order to validate the results obtained from our 108 patients, we also examined expression of SSTRs in 27 GPAs operated on between 2010 and 2014 at the University of Tübingen and at the Technical University of Munich, Germany. Five patients were females. The median age of patients was 60 years (mean 59.5 years; range $25-81$ years). All tumors were macroadenomas and clinically nonfunctioning. Medical records were available for 17 of the patients. Six of them showed unilateral or bilateral extension into the cavernous sinus and none showed bone invasion. Ten were discovered incidentally at neuroimaging performed for other reasons, six came to medical attention due to visual defects, and one due to signs and symptoms secondary to hypopituitarism. One patient was operated for a recurrent adenoma (the primary tumor was operated on elsewhere). None of the patients received any medical treatment or radiotherapy before initial surgery.

The original H\&E-stained sections and immunostains for anterior pituitary hormones, MIB1 (Ki-67), and p53 were available for review. No samples were classified either as atypical adenoma or as oncocytoma. Two expressed $\alpha \mathrm{GSU}$ (glycoprotein hormone $\alpha$ subunit), three expressed follicle-stimulating hormone ( $\mathrm{FSH} \beta$ subunit ( $\mathrm{FSH} \beta)$ ), 11 expressed luteinizing hormone (LH $\beta$ subunit (LH $\beta)$ ), and 12 expressed combinations of the gonadotropin subunits. The Ki-67 labeling index was equal to or higher than 3\% in three cases; the tissue was positive for p53 in two cases.

These studies were approved by the ethical committees of the University of Tübingen and the Technical University of Munich, and patients signed an informed consent.

Published by Bioscientifica Ltd. 


\section{Immunohistochemistry}

Immunohistochemical staining for SSTRs was performed using an automated immunostainer (Ventana Medical Systems, Tucson, AZ, USA) as reported previously (Lee et al. 2013). The SuperSentitive IHC detection system from BioGenex (Fremon, CA, USA) was used to visualize the antibody binding following the manufacturer's instructions. Sections were counterstained with Mayer's Hemalum, dehydrated, and coverslipped. The primary antibodies directed against SSTR2 (clone UMB-1 reacting with the SSTR2a isoform, dilution: 1/500), SSTR3 (clone UMB-5, dilution: 1/750), and SSTR5 (clone UMB-4, dilution: 1/75) were purchased from Abcam (Cambridge, MA, USA). Sections of normal pancreas were used as a positive control and included in each run. Sections incubated without the primary antibody were included in each batch as a negative control.

\section{Evaluation of immunostains for SSTRs}

Immunostains were evaluated semi-quantitatively on acquired images. An immunoreactive score (IRS) was recorded for each section. The IRS was generated noting the intensity of the staining (no staining, 0; mild, 1; moderate, 2; and strong, 3) and the percentage of cells showing membranous or cytoplasmic expression (no positive cells, $0 ;<10 \%$ of positive cells, $1 ; 10-50 \%$ of positive cells, $2 ; 51-80 \%$ of positive cells, 3 ; and $>80 \%$ of positive cells, 4). The overall IRS was calculated as (percentage of positive cells) $\times$ (intensity of staining). We considered the staining as being negative for IRSs 0 and 1 , weakly positive for IRSs 2 and 3, moderately positive for IRSs $4-8$, and strongly positive for IRSs $>8$. The slides were scored semi-quantitatively by three experienced neuropathologists, the coauthors of this study (R B, J S, and F R). Scoring was performed independently, by a double-blind method, according to the criteria reported above with an inter-observer variability ranging from $1 \%$ to $3.7 \%$. Discrepancies were discussed among the three pathologists.

\section{Statistical analysis}

A paired two-tailed Student's $t$-test was used to detect significant differences between two series of data and $P$ value $<0.05$ was considered significant. To compare the distribution of IRSs between matched primary and recurrent samples, we performed the Mann-Whitney $U$ paired test, and the differences were taken to be statistically significant at $P<0.05$.

\section{Results}

\section{Test patient cohort}

The median age of the 108 GPA patients included in this study was 56 years (mean 56 years; range $24-84$ years). Of the total number of patients, 35 were female; 82 patients $(75.9 \%)$ came to medical attention with visual field defects secondary to chiasm compression. Nine adenomas were discovered incidentally at neuroimaging performed for other causes; one occurred in a patient with MEN1. Two female patients had a functioning GPA and both presented with amenorrhea secondary to abnormal FSH secretion. Twenty-two patients had signs and symptoms of hypopituitarism and seven presented with headache; two patients had reduced libido, four had amenorrhea, and one gynecomastia secondary to high prolactin.

All tumors were macroadenomas. Twenty-seven cases showed uni- or bilateral extension into the cavernous sinus and 14 invaded the sellar floor (altogether 37.9\%); of the bone-invasive lesions, four also extended into the cavernous sinus.

None of the patients received any medical treatment or radiotherapy before initial surgery. Eight were irradiated after the initial TSS to treat the residual adenoma and 11 had radiotherapy after subsequent radiological evidence of recurrent disease (altogether 19/108, 17.6\%).

The clinicopathological characteristics of patients are summarized in Table 1.

\section{SSTR expression in GPAs}

SSTR2 and SSTR3 were expressed in 25.4 and $94.06 \%$ of the GPAs respectively. SSTR2 showed membranous expression, whereas SSTR3 showed both a membranous and cytoplasmic localization (Fig. 1A), as reported previously (Lupp et al. 2012). SSTR5 was expressed in two cases. Normal pancreas showed membranous expression of the three receptors. The mean IRS for SSTR2 in GPAs was $1.4 \pm 2.5$, with $74.6 \%(88 / 118)$ of the samples showing IRSs 0-1 (scored negative), and 16\% (19/118) displaying moderate to strong immunoreactivity (IRS $\geq 4$ ) (Fig. 1B). The mean IRS for SSTR3 was $7 \pm 3.45$, with $83 \%(98 / 118)$ of the adenomas having moderate to high expression levels (IRS $\geq 4$ ) (Fig. 1B). SSTR3 was expressed significantly more than SSTR2 $(P=7.29037 \times$ $10^{-45}$ ) in GPAs. We looked for possible associations between expression of SSTR3 and invasion, recurrence, or residual disease and found no significant correlation.

Published by Bioscientifica Ltd. 
A

IRS 2-3

IRS 4-8

IRS 9-12
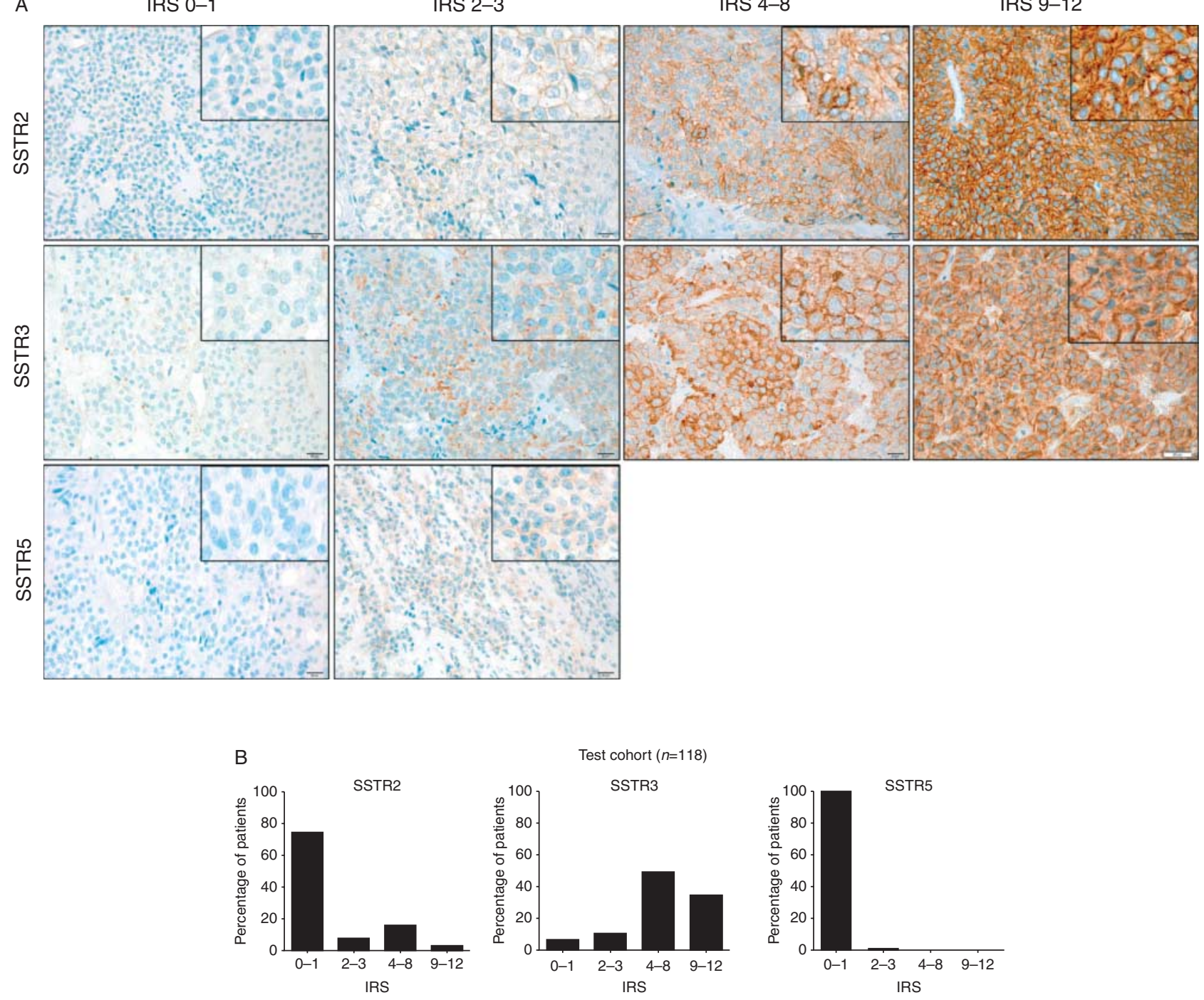

C

Validation cohort $(n=27)$
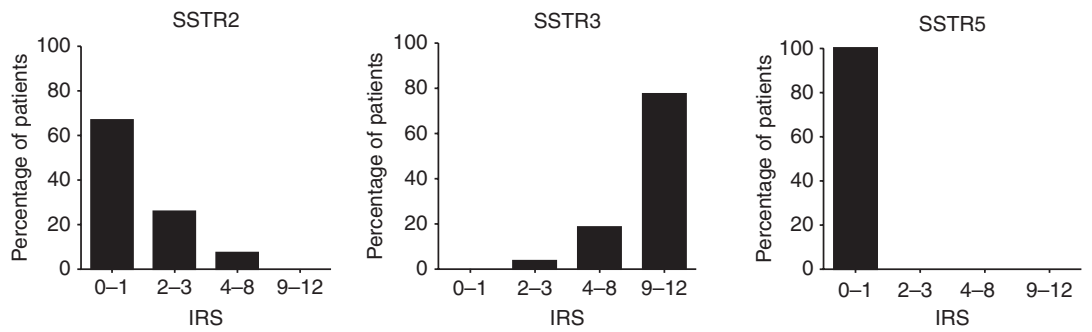

\section{Figure 1}

Expression of SSTR2, SSTR3, and SSTR5 in GPAs. (A) Immunohistochemical staining for SSTR2, SSTR3, and SSTR5 in representative GPA cases (immunoperoxidase, $\times 200$; insets $\times 400$ ) (scale bars $=20 \mu \mathrm{m}$ ).

Expression of SSTR3 in atypical and grade $2 \mathrm{~b}$ adenomas was high (mean IRS $9 \pm 2.35$ ). The full-length SSTR5 was only weakly expressed (IRS 2) in two adenomas (Fig. 1B).
(B) Immunoreactive scores (IRSs) for expression of SSTR2, SSTR3, and SSTR5 in our test cohort of 118 GPA samples. (C) IRSs for expression of SSTR2, SSTR3, and SSTR5 in our validation cohort of 27 independent GPA samples.

\section{SSTR expression in tumor recurrence}

Of the 108 patients, $18(16.7 \%)$ underwent a second operation for recurrent disease. The primary tumor was

Published by Bioscientifica Ltd 
A

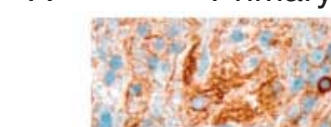

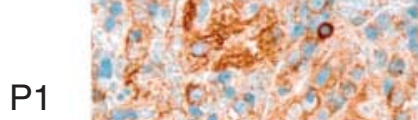

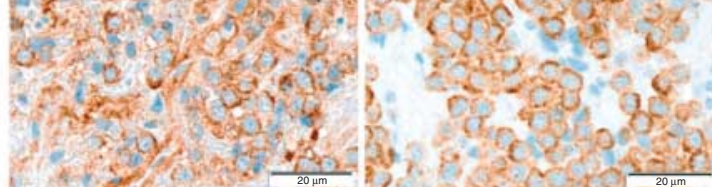

P2
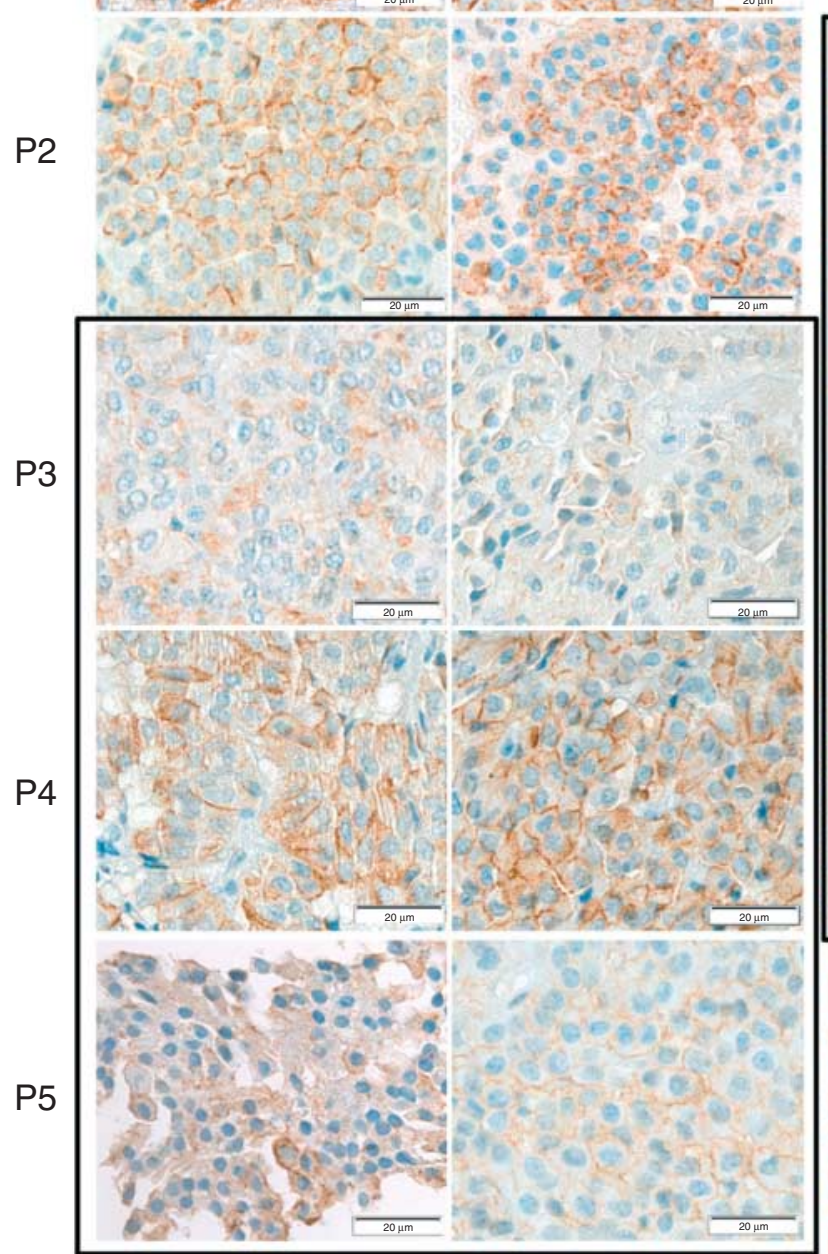

Primary
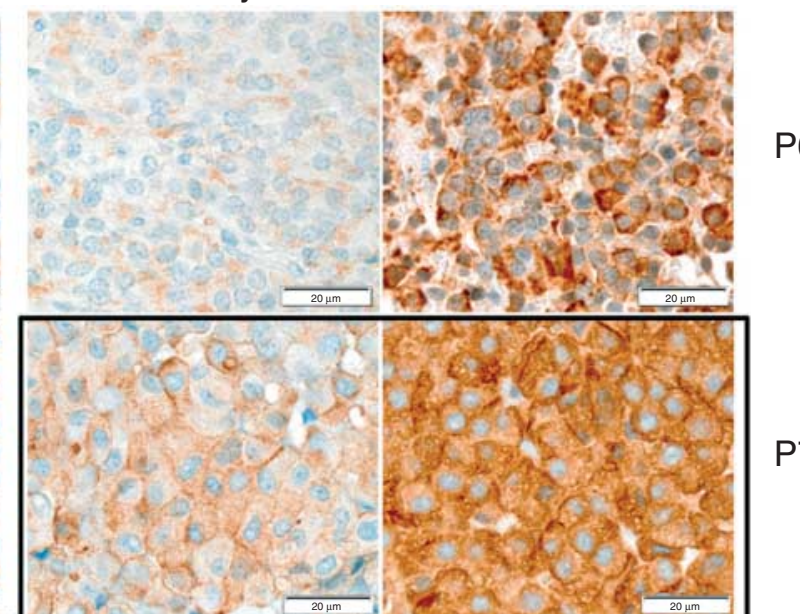

P7

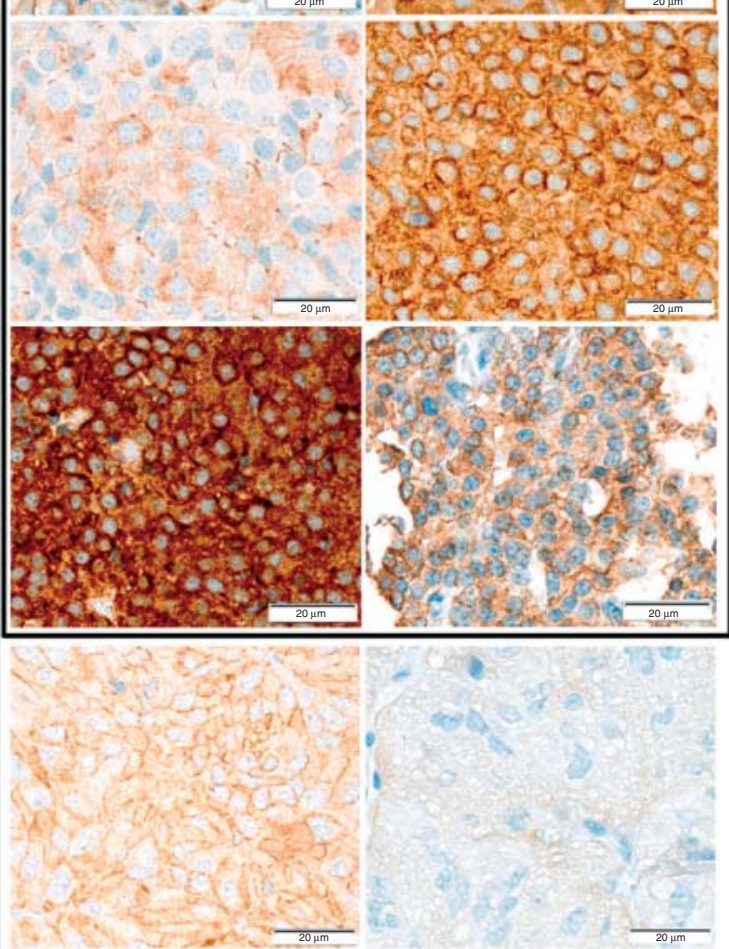

B

\begin{tabular}{|c|c|c|c|c|c|c|}
\hline \multirow[t]{2}{*}{ Patient ID } & \multicolumn{2}{|c|}{ IRS SSTR2 } & \multicolumn{2}{|c|}{ IRS SSTR3 } & \multicolumn{2}{|c|}{ Ki-67 (\%) } \\
\hline & Primary & Recurrence & Primary & Recurrence & Primary & Recurrence \\
\hline $\mathrm{P} 1$ & 0 & 1 & 6 & 6 & $<1$ & 2 \\
\hline $\mathrm{P} 2$ & 0 & 0 & 4.5 & 6 & 2 & $7-8$ \\
\hline P3 & 1 & 1 & 3 & 3 & $<1$ & $2-3$ \\
\hline P4 & 0 & 2 & 8 & 8 & $<1$ & $<1$ \\
\hline P5 & 3 & 4 & 10 & 10 & $<1$ & 1 \\
\hline P6 & 0 & 0 & 2 & 10 & $<1$ & $<1$ \\
\hline P7 & 2 & 3 & 8 & 12 & $<1$ & 1 \\
\hline P8 & 0 & 4 & 6 & 12 & $<1$ & 3 \\
\hline $\mathrm{P9}$ & 0 & 0 & 12 & 8 & $<1$ & $1-2$ \\
\hline P10 & 0 & 2 & 6 & 2 & $<1$ & $<1$ \\
\hline
\end{tabular}


available for ten patients; six of them underwent radiotherapy after the first surgery. Tumors that recurred showed a SSTR profile similar to the other GPAs. The IRS for SSTR2 slightly increased in the recurrent samples $(P=0.031)$. The IRS for SSTR3 in ten paired primaryrecurrent lesions was similar in four cases, increased in the recurrence in four cases, and decreased in two recurrences $(P=0.376)$ (Fig. $2 \mathrm{~A}$ and $\mathrm{B})$. Following radiotherapy, the IRS for SSTR3 did not change or increased in five recurrent samples, while it decreased in only one case to IRS 8 .

\section{SSTR expression in the independent validation series}

To validate our results, we investigated the expression of SSTR2, SSTR3, and SSTR5 in a series of 27 GPAs collected at two different institutions. The mean IRS for SSTR2 in the validation series was $1.04 \pm 1.47$, with $66.7 \%(18 / 27)$ of the samples showing IRSs 0-1 (scored negative) and 7.4\% (2/27) displaying moderate to strong immunoreactivity (IRS $\geq 4$ ) (Fig. 1C). The mean IRS for SSTR3 was $10 \pm 2.25$ and $96.3 \%(26 / 27)$ of the adenomas showed moderate to high expression levels (IRS $\geq 4$ ) (Fig. 1C). SSTR3 was expressed significantly more than SSTR2 $(P=1.49947 \times$ $\left.10^{-21}\right)$. No samples in the validation cohort showed immunoreactivity for SSTR5.

Remarkably, GPA samples collected at different centers (validation cohort) showed a SSTR expression profile virtually identical to that of our test cohort.

\section{Discussion}

Using validated MABs (Korner et al. 2012, Lupp et al. 2012, Gatto et al. 2013), we have assessed the expression of SSTR2, SSTR3, and SSTR5 in a cohort of 118 clinically and pathologically well-characterized primary and recurrent GPAs. Results were validated in an independent cohort of 27 GPAs operated on at different institutions. In our test cohort, we have demonstrated moderate to high expression of SSTR3 in $83 \%$ of cases, while only $16 \%$ of them showed moderate to strong immunoreactivity for SSTR2. Importantly, highly similar results were obtained from analysis of our validation cohort of GPA samples. Indeed, in this series, moderate to high expression of
SSTR3 was observed in $96.4 \%$ of cases, whereas only $7.4 \%$ of them displayed moderate to strong immunoreactivity for SSTR2.

Full-length SSTR5 was virtually absent in both sample series. Splice variants of the human SSTR5 gene have been found in pituitary adenomas (Duran-Prado et al. 2009), but the encoded truncated isoforms cannot be detected by the anti-SSTR5 antibody we used, which is directed against the C-terminal region of the receptor. SSTR1 expression was investigated in 40 adenomas of the test cohort, including the recurrences, and in 10 tumors of the validation cohort. As we observed weak, diffuse expression (IRSs 2-3) in 11 out of 50 samples, while the remaining cases were virtually negative (Supplementary Figure 2, see section on supplementary data given at the end of this article), SSTR1 was not investigated further.

Previous studies investigating the levels of mRNA or protein of SSTRs in NFPAs/GPAs have produced conflicting results. Using polyclonal anti-SSTRs antibodies, Pawlikowski et al. (2003) reported high expression of SSTR1, SSTR2, and SSTR5, with only little or no expression of SSTR3 in 13 GPAs and five null cell adenomas. The same group later found SSTR3 to be the most commonly expressed subtype in GPAs, followed by SSTR2, with no expression of SSTR5 (Pisarek et al. 2009). More recently, Ramirez et al. (2012) have examined expression of SSTRs on tissue microarrays from 74 NFPAs using the same MABs as used our study. They observed SSTR2 to be the most prevalent receptor subtype (expressed in $60 \%$ of cases), while immunopositivity for both SSTR3 and SSTR5 was observed in approximately $45 \%$ of adenomas. Different scoring criteria (membranous versus cytoplasmic staining of SSTR2) and/or the size of tissue samples (whole sections versus cores) may explain the discrepancy between results obtained by us and those obtained by Ramirez et al. (2012). A study on 12 NFPAs, five of which recurred, confirms our observation of a tendency toward higher levels of SSTRs in the recurrent lesions compared with the corresponding primary adenoma (Pisarek et al. 2011).

In tumor cells, upon ligand binding, SSTR3 is known to inhibit mitogenic pathways by activating protein tyrosine phosphatases and ultimately inactivating Raf1 and MAPK (Theodoropoulou \& Stalla 2013). SSTR3 also induces apoptosis by activating p53 and caspases, and

\section{Figure 2}

Expression of SSTRs in paired primary-recurrent tumor samples. (A) Immunohistochemical staining for SSTR3 in the matched primaryrecurrent samples. Patient ID $(P)$ is shown at the side. The boxed samples come from patients who received radiotherapy after the first surgery.
(B) Table summarizing the IRSs for SSTR2 and SSTR3, and Ki-67 positivity of the paired primary-recurrent samples. Patients indicated in blue are those who received radiotherapy after the first surgery. These samples were negative for SSTR5. 
represses endothelial cell proliferation (Florio et al. 2003, Theodoropoulou \& Stalla 2013). Thus, targeting SSTR3 might induce cytostatic and cytotoxic effects, as well as inhibiting angiogenesis. The evidence of elevated expression of SSTR3 in potentially aggressive adenomas (atypical or grade $2 \mathrm{~b}, 6 \%$ in our test cohort), and in all but two recurrent lesions (including those that recurred after irradiation), supports the rationale of targeting SSTR3 in patients with aggressive/recurrent GPAs. Among the currently available SSAs, pasireotide represents the most promising compound, given its 11-fold higher functional activity on SSTR3 than octreotide and lanreotide. Evidence obtained during in vitro studies that pasireotide inhibits the viability of NFPAs in primary cultures (Zatelli et al. 2007) further supports the hypothesis that SSTR3 is a suitable target for treatment. Indeed, the anti-proliferative effect of pasireotide on NFPAs cultures, in addition to a suppressive action on VEGF secretion (Zatelli et al. 2007), may also be mediated by pathways downstream of SSTR3.

Similar to a previous series (Young et al. 1996), approximately $80 \%$ of the patients in our test cohort presented with visual impairment secondary to chiasm compression. TSS achieved chiasm decompression in all but eight patients who required post-operative radiotherapy to treat residual tissue still encroaching on the chiasm. Octreotide and lanreotide can cause shrinkage of a substantial number of somatotroph and thyrotroph adenomas, while clinical data on pasireotide are limited to a few studies. Petersenn et al. (2010) obtained a $>20 \%$ reduction in tumor volume in $39 \%$ in acromegalic patients, and Colao et al. (2012) observed a reduction of up to $43.8 \%$ in tumor volume in patients with Cushing's disease treated with pasireotide. To date, there are no published studies on the efficacy of pasireotide in GPA patients, as these patients are just now being recruited in an ongoing clinical trial. The results of this trial will help to determine whether pasireotide can induce tumor shrinkage in GPAs.

Two of the patients had functioning GPAs (FSHsecreting), both showing high levels of expression of SSTR3 (IRSs 6 and 12). These tumors are uncommon. They usually occur in women of reproductive age presenting with menstrual irregularities, infertility, or ovarian hyperstimulation syndrome. Functioning GPAs are difficult to treat medically when surgery fails to completely remove the adenoma (Mor et al. 2005, Ntali et al. 2014). Our evidence of high levels of SSTR3 in these tumors, and the fact that SSTR3 has an anti-secretory action (Eigler et al. 2014) similar to other SSTRs, may help to guide the medical therapy of such patients.
In conclusion, we show that SSTR3 is the predominant SSTR in GPAs. Our results may explain the limited efficacy of octreotide and lanreotide in patients with this type of adenoma and provide the rationale for investigating the effect of alternative SSAs with a higher affinity for SSTR3 (such as pasireotide) in GPAs, especially in patients with large invasive or recurrent adenomas. Our data underpin the utility of profiling for SSTRs to stratify patients with pituitary adenomas for therapy with SSAs.

\section{Supplementary data}

This is linked to the online version of the paper at http://dx.doi.org/10.1530/ ERC-14-0472.

\section{Declaration of interest}

The authors declare that there is no conflict of interest that could be perceived as prejudicing the impartiality of the research reported.

\section{Funding}

Financial support for this project was provided by grant SFB824, subproject B08, from the Deutsche Forschungsgemeinschaft (DFG) and grant number 110874 from the Deutsche Krebshilfe to N S Pellegata.

\section{Acknowledgements}

The authors thank E Samson for help in cutting the tissue blocks. Tissue samples of the main cohort were provided by the Imperial College Healthcare NHS Trust Tissue Bank. Other investigators may have received samples from the same tissues.

\section{References}

Ben-Shlomo A \& Melmed S 2008 Somatostatin agonists for treatment of acromegaly. Molecular and Cellular Endocrinology 286 192-198. (doi:10.1016/j.mce.2007.11.024)

Berkmann S, Schlaffer S, Nimsky C, Fahlbusch R \& Buchfelder M 2014 Follow-up and long-term outcome of nonfunctioning pituitary adenoma operated by transsphenoidal surgery with intraoperative high-field magnetic resonance imaging. Acta Neurochirurgica 156 2233-2243. (doi:10.1007/s00701-014-2210-x)

Brochier S, Galland F, Kujas M, Parker F, Gaillard S, Raftopoulos C, Young J, Alexopoulou O, Maiter D \& Chanson P 2010 Factors predicting relapse of nonfunctioning pituitary macroadenomas after neurosurgery: a study of 142 patients. European Journal of Endocrinology 163 193-200. (doi:10.1530/EJE-10-0255)

Colao A, Grasso LF, Pivonello R \& Lombardi G 2011 Therapy of aggressive pituitary tumors. Expert Opinion on Pharmacotherapy 12 1561-1570. (doi:10.1517/14656566.2011.5)

Colao A, Petersenn S, Newell-Price J, Findling JW, Gu F, Maldonado M, Schoenherr U, Mills D, Salgado LR, Biller BM et al. 2012 A 12-month phase 3 study of pasireotide in Cushing's disease. New England Journal of Medicine 366 914-924. (doi:10.1056/ NEJMoa1105743)

Published by Bioscientifica Ltd 
Cuevas-Ramos D \& Fleseriu M 2014 Somatostatin receptor ligands and resistance to treatment in pituitary adenomas. Journal of Molecular Endocrinology 52 R223-R240. (doi:10.1530/JME-14-0011)

Duran-Prado M, Gahete MD, Martinez-Fuentes AJ, Luque RM, Quintero A, Webb SM, Benito-Lopez P, Leal A, Schulz S, Gracia-Navarro F et al. 2009 Identification and characterization of two novel truncated but functional isoforms of the somatostatin receptor subtype 5 differentially present in pituitary tumors. Journal of Clinical Endocrinology and Metabolism 94 2634-2643. (doi:10.1210/jc.2008-2564)

Eigler T, Ben-Shlomo A, Zhou C, Khalafi R, Ren SG \& Melmed S 2014 Constitutive somatostatin receptor subtype-3 signaling suppresses growth hormone synthesis. Molecular Endocrinology 28 554-564. (doi:10.1210/me.2013-1327)

Florio T, Morini M, Villa V, Arena S, Corsaro A, Thellung S, Culler MD, Pfeffer U, Noonan DM, Schettini G et al. 2003 Somatostatin inhibits tumor angiogenesis and growth via somatostatin receptor-3-mediated regulation of endothelial nitric oxide synthase and mitogen-activated protein kinase activities. Endocrinology 144 1574-1584. (doi:10.1210/ en.2002-220949)

Gatto F, Feelders RA, van der Pas R, Kros JM, Waaijers M, Sprij-Mooij D, Neggers SJ, van der Lelij AJ, Minuto F, Lamberts SW et al. 2013 Immunoreactivity score using an anti-sst2A receptor monoclonal antibody strongly predicts the biochemical response to adjuvant treatment with somatostatin analogs in acromegaly. Journal of Clinical Endocrinology and Metabolism 98 E66-E71. (doi:10.1210/jc.2012-2609)

Grozinsky-Glasberg S, Shimon I, Korbonits M \& Grossman AB 2008 Somatostatin analogues in the control of neuroendocrine tumours: efficacy and mechanisms. Endocrine-Related Cancer 15 701-720. (doi:10.1677/ERC-07-0288)

Korner M, Waser B, Schonbrunn A, Perren A \& Reubi JC 2012 Somatostatin receptor subtype $2 \mathrm{~A}$ immunohistochemistry using a new monoclonal antibody selects tumors suitable for in vivo somatostatin receptor targeting. American Journal of Surgical Pathology 36 242-252. (doi:10.1097/PAS.0b013e31823d07f3)

Lee M, Marinoni I, Irmler M, Psaras T, Honegger JB, Beschorner R, Anastasov N, Beckers J, Theodoropoulou M, Roncaroli F et al. 2013 Transcriptome analysis of MENX-associated rat pituitary adenomas identifies novel molecular mechanisms involved in the pathogenesis of human pituitary gonadotroph adenomas. Acta Neuropathologica 126 137-150. (doi:10.1007/s00401-013-1132-7)

Lloyd RJ, Kovacs K, Young WF Jr, Farrell WE, Asa SL, Trouillas J, Kontogeorgos G, Sano T, Scheithauer BW, Horvath E et al. 2004 Tumours of the pituitary gland. In Pathology and Genetics, pp 9-48. Eds RA DeLellis, RV Lloyd \& PU Heitz. Lyon: International Agency for Research and Cancer (IARC).

Lupp A, Nagel F, Doll C, Rocken C, Evert M, Mawrin C, Saeger W \& Schulz S 2012 Reassessment of sst $_{3}$ somatostatin receptor expression in human normal and neoplastic tissues using the novel rabbit monoclonal antibody UMB-5. Neuroendocrinology 96 301-310. (doi:10.1159/ 000337659)

Mor E, Rodi IA, Bayrak A, Paulson RJ \& Sokol RZ 2005 Diagnosis of pituitary gonadotroph adenomas in reproductive-aged women. Fertility and Sterility 84 757.e1-757.e6. (doi:10.1016/j.fertnstert.2005.02.050)

Nielsen S, Mellemkjaer S, Rasmussen LM, Ledet T, Olsen N, Bojsen-Moller M, Astrup J, Weeke J \& Jorgensen JO 2001 Expression of somatostatin receptors on human pituitary adenomas in vivo and ex vivo. Journal of Endocrinological Investigation 24 430-437. (doi:10.1007/BF03351043)

Ntali G, Capatina C, Grossman A \& Karavitaki N 2014 Functioning gonadotroph adenomas. Journal of Clinical Endocrinology and Metabolism 99 4423-4433. (doi:10.1210/jc.2014-2362)

Pawlikowski M, Pisarek H, Kunert-Radek J \& Radek A 2003 Immunohistochemical detection of somatostatin receptor subtypes in "clinically nonfunctioning" pituitary adenomas. Endocrine Pathology 14 231-238. (doi:10.1007/s12022-003-0015-1)

Petersenn S, Schopohl J, Barkan A, Mohideen P, Colao A, Abs R, Buchelt A, Ho YY, Hu K, Farrall AJ et al. 2010 Pasireotide (SOM230) demonstrates efficacy and safety in patients with acromegaly: a randomized, multicenter, phase II trial. Journal of Clinical Endocrinology and Metabolism 95 2781-2789. (doi:10.1210/jc.2009-2272)

Pisarek H, Pawlikowski M, Kunert-Radek J \& Radek M 2009 Expression of somatostatin receptor subtypes in human pituitary adenomas immunohistochemical studies. Endokrynologia Polska 60 240-251.

Pisarek H, Kunert-Radek J, Radek M, Swietoslawski J, Winczyk K \& Pawlikowski M 2011 Expression of somatostatin receptor subtypes in primary and recurrent gonadotropinomas: are somatostatin receptors involved in pituitary adenoma recurrence? Neuro Endocrinology Letters 32 96-101.

Ramirez C, Cheng S, Vargas G, Asa SL, Ezzat S, Gonzalez B, Cabrera L, Guinto G \& Mercado M 2012 Expression of Ki-67, PTTG1, FGFR4, and SSTR 2, 3, and 5 in nonfunctioning pituitary adenomas: a high throughput TMA, immunohistochemical study. Journal of Clinical Endocrinology and Metabolism 97 1745-1751. (doi:10.1210/jc.20113163)

Schmid HA \& Schoeffter P 2004 Functional activity of the multiligand analog SOM230 at human recombinant somatostatin receptor subtypes supports its usefulness in neuroendocrine tumors. Neuroendocrinology 80 (Suppl 1) 47-50. (doi:10.1159/000080741)

Theodoropoulou M \& Stalla GK 2013 Somatostatin receptors: from signaling to clinical practice. Frontiers in Neuroendocrinology $\mathbf{3 4}$ 228-252. (doi:10.1016/j.yfrne.2013.07.005)

Trouillas J, Roy P, Sturm N, Dantony E, Cortet-Rudelli C, Viennet G, Bonneville JF, Assaker R, Auger C, Brue T et al. 2013 A new prognostic clinicopathological classification of pituitary adenomas: a multicentric case-control study of 410 patients with 8 years postoperative follow-up. Acta Neuropathologica 126 123-135. (doi:10.1007/ s00401-013-1084-y)

Webb SM, Ware JE, Forsythe A, Yang M, Badia X, Nelson LM, Signorovitch JE, McLeod L, Maldonado M, Zgliczynski W et al. 2014 Treatment effectiveness of pasireotide on health-related quality of life in patients with Cushing's disease. European Journal of Endocrinology 171 89-98. (doi:10.1530/EJE-13-1013)

Young WF Jr, Scheithauer BW, Kovacs KT, Horvath E, Davis DH \& Randall RV 1996 Gonadotroph adenoma of the pituitary gland: a clinicopathologic analysis of 100 cases. Mayo Clinic Proceedings $\mathbf{7 1}$ 649-656. (doi:10.1016/S0025-6196(11)63002-4)

Zatelli MC, Piccin D, Vignali C, Tagliati F, Ambrosio MR, Bondanelli M, Cimino V, Bianchi A, Schmid HA, Scanarini M et al. 2007 Pasireotide, a multiple somatostatin receptor subtypes ligand, reduces cell viability in non-functioning pituitary adenomas by inhibiting vascular endothelial growth factor secretion. Endocrine-Related Cancer 14 91-102. (doi:10.1677/ERC-06-0026)

Received in final form 4 December 2014

Accepted 16 December 2014

Made available online as an Accepted Preprint

16 December 2014 http://erc.endocrinology-journals.org DOI: 10.1530/ERC-14-0472 (c) 2015 Society for Endocrinology Printed in Great Britain 